\title{
Does the degree of polymerization of haptoglobin influence the results of the radial immunodiffusion assay?
}

\author{
H.J.M. Van Rijn ${ }^{\text {a }}$, J.P.W. Wisman ${ }^{a}$ and F. Gmelig Meyling ${ }^{b}$ \\ a Clinical and Haematological Laboratory, 'Dr. A. Mathisen Hospital' \\ and ${ }^{b}$ Department of Clinical Immunology, University Hospital, Utrecht (The Netherlands)
}

Key words: Haptoglobin; Radial immunodiffusion

\section{Summary}

The degree of polymerization of haptoglobin $(\mathrm{Hp})$ phenotypes 2-1 and 2-2 was studied. In individual samples the degree of polymerization tends to be positively correlated with the $\mathrm{Hp}$ concentration as determined by the radial immunodiffusion technique (RID), while an inverse relationship between the size of Hp polymers and the Hp concentration in RID was expected.

After reductive cleavage of $\mathrm{Hp}$, the apparent $\mathrm{Hp}$ concentration became higher in individual samples with different degrees of polymerization; the shift to higher values appeared to be independent of the degree of polymerization in samples of phenotypes Hp 2-1 and Hp 2-2.

Consequently, the degree of polymerization as such does not have an impact on the outcome of RID at a technical level.

\section{Introduction}

The diagnostic approach to haemolytic anaemia is usually performed in two steps. These are demonstrating that a haemolytic anaemia is present, followed by determining the specific cause of the condition. A number of laboratory tests are available. These include the reticulocyte count, haematocrit measurement, the test for red cell osmotic fragility, red cell survival time measured by the ${ }^{51} \mathrm{Cr}$ method, the determination of free plasma haemoglobin and haptoglobin (Hp). Work in our laboratory has been focussed on aspects of the latter two techniques.

Correspondence to: Dr. H.J.M. van Rijn, Clinical and Haematological Laboratory, Dr. A. Mathijsen Hospital, P.O. Box 90000, 3509 AA Utrecht, The Netherlands. 
Using derivative spectrophotometry we developed an assay for free haemoglobin in plasma [1]: this technique can also be successfully performed for the assessment of pigments in other body fluids [2]. Our studies on the $\mathrm{Hp}$ molecule have been primarily directed at the clinical significance of $\mathrm{Hp}$ as a laboratory parameter [3-5], and at present the role of the Hp phenotype is the object of study [6].

The serum haptoglobin ( $\mathrm{Hp}$ ) phenotypes are determined by two alleles, $\mathrm{Hp} 1$ and $\mathrm{Hp} 2$, which can combine to form the three most common phenotypes $\mathrm{Hp} \mathrm{1-1,} \mathrm{Hp}$ 2-1 and Hp 2-2 as distinguished by conventional starch gel electrophoresis or by polyacrylamide gel electrophoresis (PAGE).

In its basic form $\mathrm{Hp}$ consists of 4 amino-acid chains: two $\alpha$-chains and two $\beta$-chains. The $\beta$-chains are invariable, but two main genetic types of the $\alpha$-chain exist, producing three phenotypes; Hp 1-1 with only $2 \alpha_{2}$-chains; Hp 2-2 with only two $\alpha_{1}$-chains, and the hybridic form $\mathrm{Hp} \mathrm{2-1.} \mathrm{Since} \mathrm{an} \alpha_{2}$-chain has two binding sites for other $\alpha$-chains, as distinct from an $\alpha_{1}$-chain which has only binding site, polymerization is possible in the case of the $\mathrm{Hp} \mathrm{2-1}$ and $\mathrm{Hp} \mathrm{2-2}$ phenotypes [5].

The phenotype Hp 1-1 produces a protein which appears homogeneous by electrophoretic mobility and ultracentrifugal sedimentation pattern. The phenotype Hp 2-2 produces a series of proteins of regularly increasing molecular size which are believed to be serial polymers of a basic unit. The protein pattern in phenotype $\mathrm{Hp}$

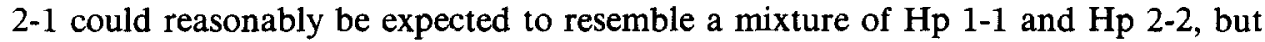
in fact, it differs from such a mixture in having polymer bands with a PAGE mobility which is different from the PAGE mobility of $\mathrm{Hp} \mathrm{2-1} \mathrm{and} \mathrm{also} \mathrm{the}$ ultracentrifugal sedimentation characteristics were different from type Hp 2-1.

Two approaches are available for the assay of haptoglobin. These are the determination of haemoglobin binding capacity in serum and the reactivity with anti-haptoglobin antisera. In the latter the antiserum used must react equally well with haptoglobin of all phenotypes. Because the relative concentrations of $\mathrm{Hp}$ components in the PAGE patterns of individual patients clearly differ due to the formation of polymers, the question arose whether the degree of polymerization influences the total amount of $\mathrm{Hp}$ as determined with RID. To our knowledge, this problem is still unresolved and is therefore the aim of this study.

\section{Materials and methods}

\section{Reagents and equipment}

Disc electrophoresis was performed using the Desaga System Havana (C. Desaga $\mathrm{GmbH}, \mathrm{FRG}$ ) on vertical polyacrylamide gels. Acrylamide was obtained from Serva (Heidelberg, FRG) $N, N^{\prime}$-methylenebisacrylamide and $N, N, N^{\prime}, N^{\prime}$-tetramethylenediamine were purchased from Eastman Kodak Co. (Rochester, NY, USA). All other chemicals were of analytical grade and were obtained from E. Merck AG (Darmstadt, FRG) or J.T. Baker Chemicals (Deventer, The Netherlands).

\section{Methods}

Mancini's method [7] was used for the radial immunodiffusion (RID) technique. Individual samples of serum were classified according to haptoglobin type by PAGE 
TABLE I

Mol wt of haptoglobin phenotype 2-1

\begin{tabular}{lrrlll}
\hline $\begin{array}{l}\text { Phenotype } \\
\text { Hp 2-1 }\end{array}$ & $\begin{array}{l}\text { Mol wt } \\
\text { (theoretical) of } \\
\text { components }\end{array}$ & \begin{tabular}{l} 
Samples taken arbitrarily \\
\cline { 4 - 5 }
\end{tabular} & $\begin{array}{l}\text { \% of each } \\
\text { component }\end{array}$ & $\begin{array}{l}\text { Contribution } \\
\text { to average mol wt }\end{array}$ & $\begin{array}{l}\text { Weighted } \\
\text { average } \\
\text { mol wt }\end{array}$ \\
\hline$\left(\alpha_{1} \beta\right)_{2}\left(\alpha_{2} \beta\right)_{n}$ & $n=0$ & 98000 & 28.2 & 27636 & \\
& 1 & 155000 & 27.4 & 42501 & \\
& 2 & 212000 & 19.1 & 40577 & \\
3 & 269000 & 11.9 & 32011 & \\
4 & 326000 & 7.9 & 25656 & \\
& 5 & 383000 & 5.5 & 21027 & \\
& & &
\end{tabular}

${ }^{\text {a }}$ Based on mol wt of the chains [10] $\alpha_{1}=9000$ daltons, $\alpha_{2}=17000$ daltons, $\beta=40000$ daltons.

b Gel scanning data of an arbitrarily taken, sample obtained as described in 'Materials and Methods'.

as described previously [3], using a reaction which detects the peroxidase-like activity of the haptoglobin-haemoglobin complexes which is only indirectly a measure of the haptoglobin concentration. However, scanning of the faint coloured haptoglobin-haemoglobin complexes appeared less reliable for quantitative results than scanning the same pattern after staining with amido-black. It appeared that when electrophoresis took place after partial purification of haptoglobin under those conditions patterns stained directly for protein with amido-black were comparable with those stained for peroxidase activity, indicating that under these conditions staining for protein directly with amido-black reflect the haptoglobin concentration in the individual components of polyacrylamide gel patterns. Therefore, routinely for the determination of the relative concentrations of the individual haptoglobin components after electrophoresis of partial purified haptoglobin in serum samples, the gels were stained with amido-black. The densitometric quantitation of the electrophoretic zones was performed with an IL Densitometer 677 (Instrumentation Laboratory Boskamp GmbH, 5303 Hersel, FRG). It was made certain that reductive cleavage was complete by controlling whether the characteristic bands in the electrophoretic pattern were disappeared. To calculate the mean relative molecular mass of haptoglobins in the various samples we used the figures of the relative molecular mass for the $\alpha_{1}$-chains, $\alpha_{2}$-chains and $\beta$-chains presented in Table I and subunit composition $\left(\alpha_{2} \beta\right)_{n}$ for $\mathrm{Hp} \mathrm{2-2}$ and $\left(\alpha_{1} \beta\right)_{2}\left(\alpha_{2} \beta\right)_{n}$ for Hp 2-1 [8], together with the relative concentrations of the individual $\mathrm{Hp}$ components.

\section{Samples}

Blood samples were collected and allowed to clot at room temperature. They were centrifuged (10 min at $1500 \times g$ at room temperature) and the serum stored. Serum samples were from healthy volunteers, from patients with high levels of acute phase proteins and from patients with suspected haemolysis.

\section{Partial haptoglobin purification}

The purification was essentially performed as described by Smithies et al [8]. Four millilitres of a 1\% suspension of DEAE A-50 Sephadex in distilled water were 
poured into a $25-\mathrm{ml}$ beaker. Distilled water, $5 \mathrm{ml}$, was added, followed by $0.4 \mathrm{ml}$ of $1.0 \mathrm{mmol} / 1$ sodium acetate $(\mathrm{pH} 4.5)$ and $1 \mathrm{ml}$ serum. The contents of the beaker was mixed for $5 \mathrm{~min}$ and then poured into a column $(10 \times 0.5 \mathrm{~cm})$. The column was allowed to drain, and washed with $25 \mathrm{ml}$ distilled and allowed to drain again.

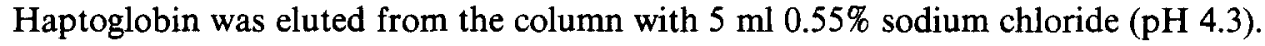
Fractions of $1 \mathrm{ml}$ were collected. The eluates were analysed for haptoglobin with the Technicon RA-1000 analyzer (Technicon Inc., Tarrytown, NY, USA) [6]. The eluates which were considered to be sufficiently rich in haptoglobin were pooled and transferred into dialysis bags. Concentration was performed by placing the dialysis bags in solid sucrose at $0^{\circ} \mathrm{C}$ for some hours. After concentration and subsequent dialysing against several changes of distilled water the preparation was ready for further experiments.

\section{Reductive cleavage}

To $0.5 \mathrm{ml}$ serum was added $25 \mu 1500 \mathrm{mmol} / 1$ boric acid $/ 200 \mathrm{mmol} / 1$ sodium hydroxide buffer, $1 \mathrm{ml}$ urea solution $(0.6 \mathrm{~g} \mathrm{ml})$ and $5 \mu 1$ of mercaptoethanol.

After 30 min gently rotating at room temperature, dissociation of the polypeptide chains $(\alpha$ and $\beta$ ) was checked by PAGE. The $\alpha$ - and $\beta$-chains did not lose their ability to react with anti-haptoglobin antisera, which was confirmed by performing the RID procedure after reductive cleavage. For each sample this process was repeated.

\section{Results and discussion}

The Hp 1-1 molecule $\left(\alpha_{1} \beta\right)_{2}$ migrates as a single electrophoretic moiety. The multiple components in $\mathrm{Hp} \mathrm{2-2} \mathrm{and} \mathrm{Hp} \mathrm{2-1}$ are a series of proteins of increasing size that are resolved by molecular sieving.

Evidence has been presented [7], that in $\mathrm{Hp} 2-2$ the single protein can link up to form linear polymers $\left(\alpha_{2} \beta\right)_{n}$ with $n=3,4,5,6,7,8$. In Hp 2-1 the assembly is more complex, both $\alpha_{1} \beta$ and $\alpha_{2} \beta$ subunits being involved [8]. In this phenotype the polymers are assembled according the formula $\left(\alpha_{1} \beta\right)_{2}\left(\alpha_{2} \beta\right)_{n}$ with $n=0,1,2,3,4$, 5 .

Consequently, both $\mathrm{Hp} \mathrm{2-2} \mathrm{and} \mathrm{Hp} 2-1$ occur as a mixture of polymers which was clearly apparent from the results obtained by PAGE scanning on individual sera. This raises the question whether the degree of polymerization would negatively influence the accessibility of some of the antigenic determinants present in the $\mathrm{Hp}$ polymers. In order to answer the question whether haptoglobin polymerization would be related to the apparent amount of haptoglobin as assessed by RID, we determined the average relative molecular mass of the haptoglobin present in individual sera. Relative concentrations of the individual polymers were estimated by means of densitometry after PAGE. The molecular mass were inferred from the position of the bands in relation with the theoretical compositions of possible polymers.

Thirty-nine different sera of phenotypes Hp 2-1, and 32 of type Hp 2-2 were submitted to PAGE followed by staining and densitometry. For phenotype $\mathrm{Hp}$ 1-1 the procedure was not performed because in the $\mathrm{Hp} \mathrm{1-1}$ pattern all of the 


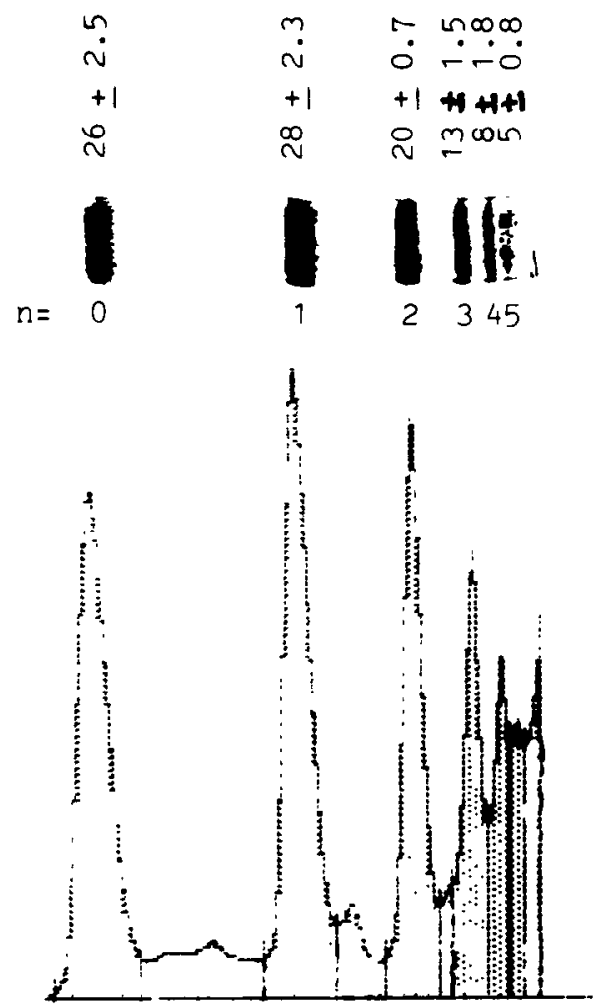

Fig. 1. A typical pattern of partial purified $\mathrm{Hp}$ with phenotype $\mathrm{Hp} 2-1$ after PAGE and staining for protein (upper part). The protein bands closely parallel the haptoglobin pattern. Scanning of protein bands was performed after PAGE. In the scanning pattern furthermore relative concentrations and standard deviations are presented. Polymers are assembled according the formula $\left(\alpha_{1} \beta\right)_{2}\left(\alpha_{2} \beta\right)_{n}$ with $n=0,1,2,3,4,5$.

haptoglobin is present as a single component and therefore a good correlation can be expected between the RID value and the Hp component determined with densitometry after PAGE in serum samples with the HP phenotype 1-1.

A typical electrophoretic pattern for phenotype Hp 2-1 is shown in Fig. 1 together with the relative concentrations of the individual components (total haptoglobin $=100 \%$ ) determined by densitometric scanning; the relevant diagram is rendered as well. The values represent the average of four independent determinations. The SDs indicate that the scanning patterns are distinct and quantitatively reproducible. The bands are numbered 0 to 5 in the sequence which most easily satisfies the observed electrophoretic pattern and according the formula $\left(\alpha_{1} \beta\right)_{2}$ $\left(\alpha_{2} \beta\right)_{n}$ with $n=0,1,2,3,4,5$ [9]. The pattern for Hp 2-2, not shown here but elsewhere already once presented [3], consists of 6 haptoglobin bands at positions different from $\mathrm{Hp}$ type 2-1. Figure 2 shows the relationship between the $\mathrm{Hp} \mathrm{2-1}$ and $\mathrm{Hp} \mathrm{2-2} \mathrm{values} \mathrm{as} \mathrm{determined} \mathrm{with} \mathrm{RID} \mathrm{and} \mathrm{the} \mathrm{mean} \mathrm{molecular} \mathrm{weight} \mathrm{of} \mathrm{Hp}$ in the individual samples. 

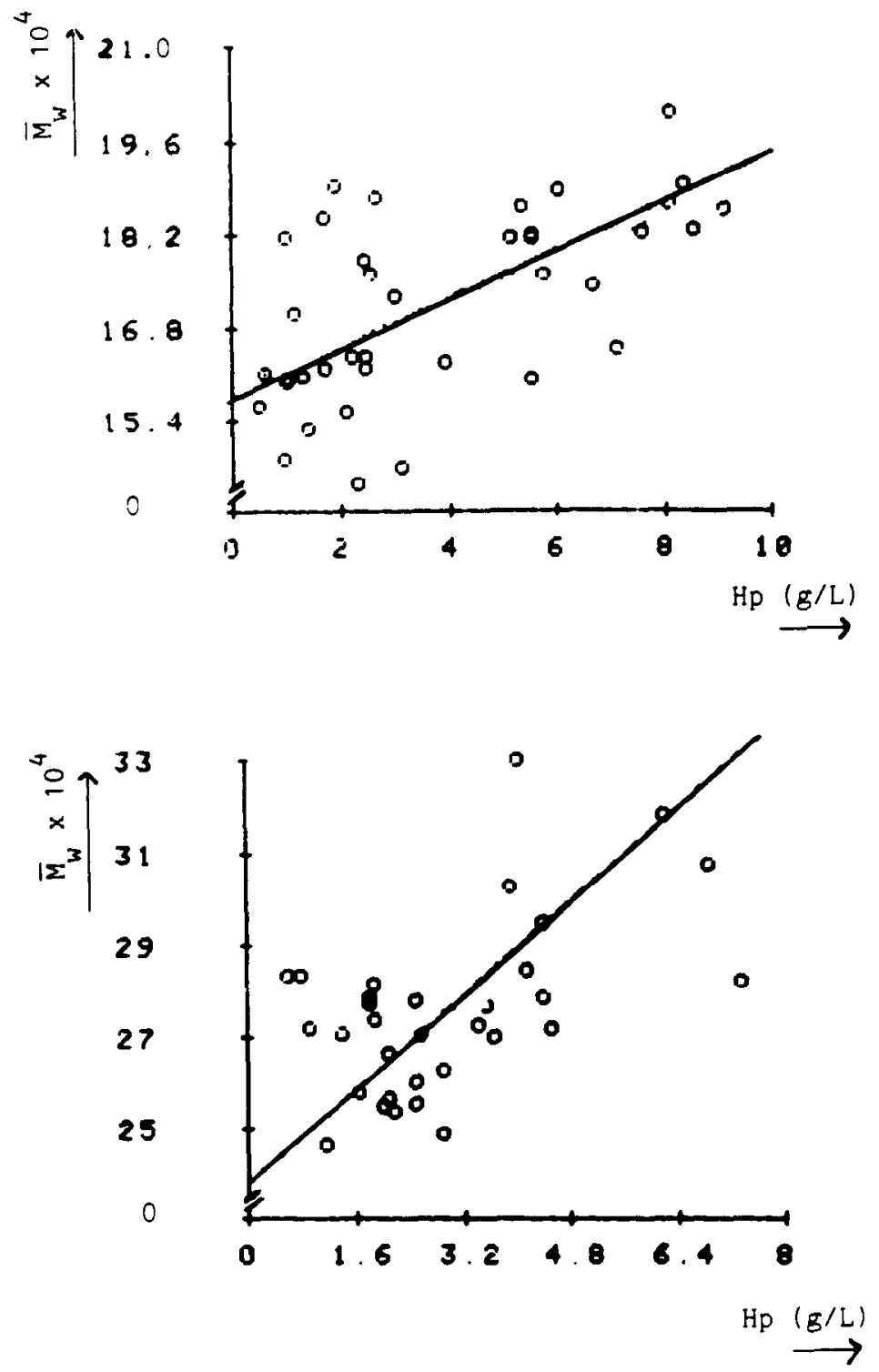

Fig. 2. The mean mol wt of $\mathrm{Hp}$ in 39 different serum samples of type Hp 2-1 with different Hp concentrations (upper part of the figure). In the lower part of the figure the mean molecular weights of 32 serum samples of type $\mathrm{Hp}$ 2-2 with different $\mathrm{Hp}$ concentrations $(\mathrm{g} / \mathrm{l})$ are presented.

For Hp 2-1 the figures of Fig. 2 are slope $=0.37$; intercept $=15.7 ; r=0.61$; $n=39 ; p<0.001$, while for Hp 2-2 the following equation holds: $y=1.27 x+23.8$, $r=0.54, n=32, p<0.01$ with $\mathrm{Hp}$ in $\mathrm{g} / 1$ taken as $x$. 
Thus, in this set of individual samples, the mean molecular weight of Hp, taken as a measure for the degree of polymerization, tends to be positively correlated with the $\mathrm{Hp}$ concentration as determined by RID.

This finding was quite surprising, because we had expected to see an inverse relationship between the size of the $\mathrm{Hp}$ polymers and the apparent $\mathrm{Hp}$ concentration in RID. The reasons for this assumption were (1) that the RID reaction rates
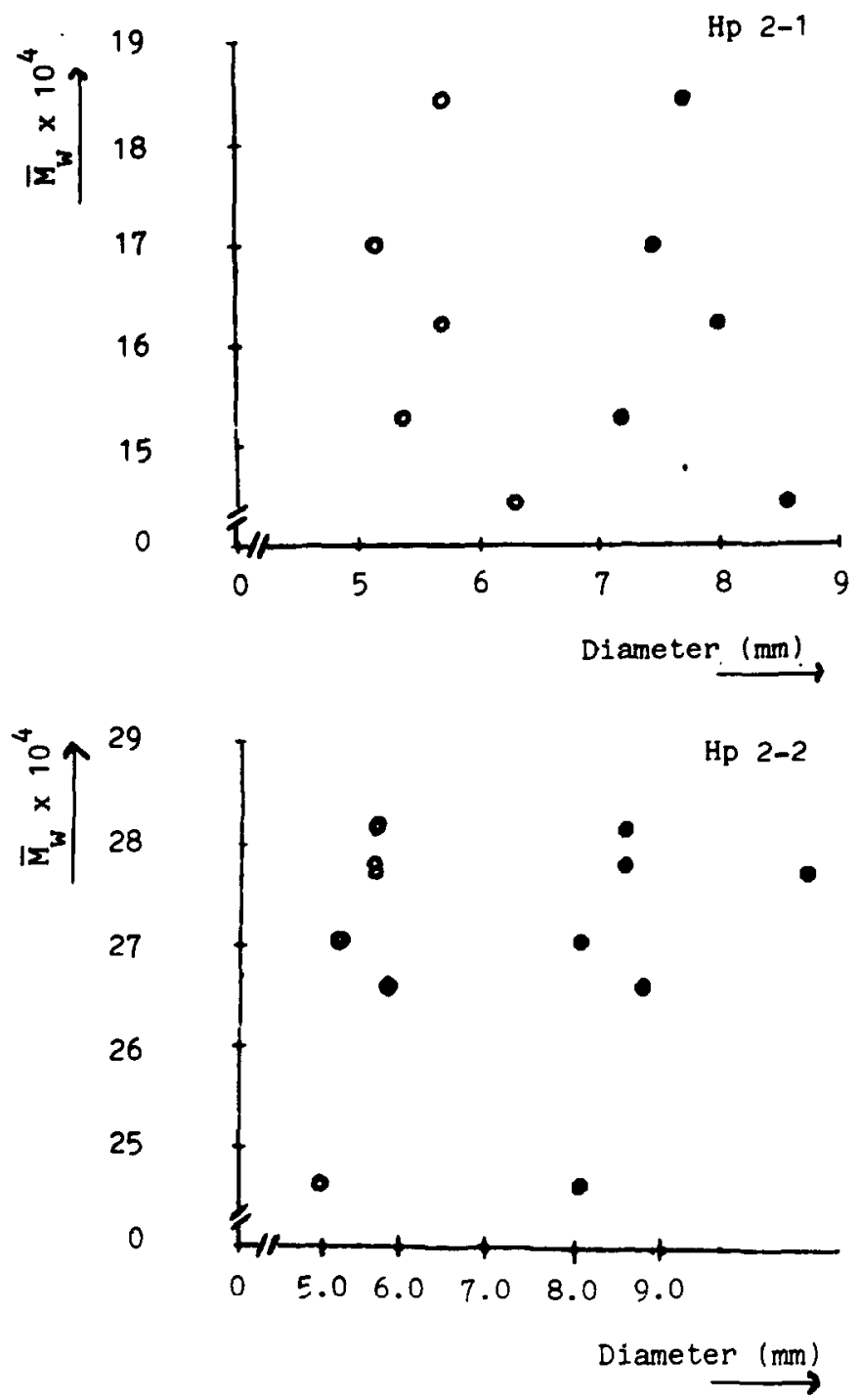

Fig. 3. Serum samples with about the same Hp concentrations, here expressed in the diameters (mm) of the precipitate rings, before, $\mathrm{O}$, and after, $\bullet$, reductive cleavage of the $\mathrm{Hp}$ molecules. Upper part samples with phenotype $\mathrm{Hp} 2-1$, lower part samples with phenotype 2-2. 
and ring diameters for $\mathrm{Hp} \mathrm{1-1,} \mathrm{Hp} \mathrm{2-1} \mathrm{and} \mathrm{Hp} \mathrm{2-2} \mathrm{are} \mathrm{partly} \mathrm{dictated} \mathrm{by} \mathrm{the} \mathrm{size} \mathrm{of}$ the molecules, particularly by the size of the largest polymeric fraction present, and (2) that a certain amount of antigenic determinations should be hidden by polymerization. Therefore, we attempted to prove the nature of the discrepancy between these theoretical considerations and our observation, by performing reductive cleavage on serum samples typed for Hp phenotype and average degree of polymerization, measuring the apparent $\mathrm{Hp}$ concentrations in intact and cleaved samples.

Figure 3 shows the results of the reductive cleavage experiment performed on eleven different serum samples containing $\mathrm{Hp}$ 2-1 or Hp 2-2. For phenotype $\mathrm{Hp}$ 2-1 5 samples with $\mathrm{Hp}$ values between 1.0 and $2.5 \mathrm{~g} / 1$ (expressed in diameters $(D)$ of the precipitate between 5.0 and $6.2 \mathrm{~mm}$ ) and different degrees of polymerization were subjected to reductive cleavage. After cleavage the apparent $\mathrm{Hp}$ concentration became higher, but no difference between the samples with a low degree and a high degree of polymerization was demonstrated; the shift to higher RID values was about constant for all samples tested. The same holds for the samples with phenotype Hp 2-2. Also here most of the samples with Hp values of about $1.7 \mathrm{~g} / 1$ (equal with a diameter of about $5.8 \mathrm{~mm}$ ) show an apparent $\mathrm{Hp}$ concentration in RID of about $5.1 \mathrm{~g} / 1$ ( $D$ is about $8.8 \mathrm{~mm}$ ) irrespective of the degree of polymerization.

Consequently, the degree of polymerization as such does not have an impact on the outcome of RID at a technical level. Thus the question remains why there is indeed a trend towards higher RID values at higher average molecular sizes of $\mathrm{Hp}$. We are trying to resolve this problem (1) by studying the behaviour of the isolated, homogenous polymeric $\mathrm{Hp}$ fractions in RID and (2) by longitudinal determination of the $\mathrm{Hp}$ polymerization rate in patients showing acute phase reactions: we are inclined to assume that the observed relationship has a biological rather than a technical basis, i.e. that an augmented $\mathrm{Hp}$ production is directly allied with an increase in the degree of polymerization. This study will be the subject of a following paper.

\section{References}

1 Sanderink GJCM, Van Rijn HJM. Quantitative measurement of plasma hemoglobin by second derivative spectrophotometry. Clin Chim Acta 1985;146:442-446.

2 Stroes JW, Van Rijn HJM. Quantitative measurement of blood pigments in cerebrospinal fluid by derivative spectrophotometry. Ann Clin Biochem 1987;124:189-197.

3 Schrijver J, Van Rijn HJM, Schreurs WHP. Re-evaluation of the haptoglobin reference values with the radial immunodiffusion technique. Clin Biochem 1984;17:258-262.

4 Van Rijn HJM, Kruit WHJ, Schrijver J. Haptoglobin typing is it clinically necessary for a reliable determination of haptoglobin with the single radial immunodiffusion technique? J Clin Chem Clin Biochem 1984;22:109-112.

5 Van Rijn HJM, Sanderink GJCM. Haptoglobin a useful laboratory parameter? Neth J Med $1986 ; 29: 16-22$.

6 Van Rijn HJM, Van der Wilt W, Stroes JW, Schrijver J. Is the turbidımetric immunoassay of haptoglobin phenotype dependent? Clin Biochem, in press.

7 Mancini G, Carbonare AD, Heremand JF. Immunochemical quantitation of antigens by single radial Immunodiffusion. Clin Chim Acta 1969;26:235-254. 
8 Smithies U, Connell GE, Dixon GH. Inheritance of haptoglobin subtypes. Am J Hum Genet 1962;14:14-21.

9 Javid J. Human Haptoglobins Curr Top Hematol 1978;1:151-192.

10 Black JA, Dixon GH. Amino acid sequences of alpha chains of human haptoglobins. Nature 1968;218:736-741. 